\title{
Zeeman-Doppler Imaging of late-type stars - The surface magnetic field of II Peg
}

\author{
T.A. Carroll *, M. Kopf , I. Ilyin \& K.G. Strassmeier \\ Astrophysikalisches Institut Potsdam, An der Sternwarte 16, D-14482 Potsdam, Germany \\ Received 11 Nov 2007, accepted 22 Nov 2007 \\ Published online later
}

Key words stars: activity, stars: magnetic fields, radiative transfer, line: formation, polarization

\begin{abstract}
Late-type stars in general possess complicated magnetic surface fields which makes their detection and in particular their modeling and reconstruction challenging. In this work we present a new Zeeman-Doppler imaging code which is especially designed for the application to late-type stars. This code uses a new multi-line cross-correlation technique by means of a principal component analysis to extract and enhance the quality of individual polarized line profiles. It implements the full polarized radiative transfer equation and uses an inversion strategy that can incorporate prior knowledge based on solar analogies. Moreover, our code utilizes a new regularization scheme which is based on local maximum entropy to allow a more appropriate reproduction of complex surface fields as those expected for late-type stars. In a first application we present Zeeman-Doppler images of II Pegasi which reveal a surprisingly large scale surface structure with one predominant (unipolar) magnetic longitude which is mainly radially oriented.
\end{abstract}

(c) 2007 WILEY-VCH Verlag GmbH \& Co. KGaA, Weinheim

\section{Introduction}

Even though the technique for Zeeman-Doppler imaging was introduced almost two decades ago by Semel (1989) there are only a very small number of magnetic surface images for late-type stars (Donati, 1999; Petit, 2006). The reason for this fact is not so much the limited number of available targets than the inherent complexity of the surface magnetism which may lead to a severe reduction or even the mutual cancellation of circular polarization profiles (Stokes $V$ ) by different magnetic polarities. The linear polarization profiles (Stokes $Q$ and $U$ ) which are, as a rule of thumb, at least one order of magnitude lower than the circular polarization are even harder to detect. Cross-correlation techniques, in its simplest form the multi-line technique of Semel \& Li (1996) or the more elaborate technique of Least-Square Deconvolution (LSD) (Donati et al., 1997) are used to extract and boost the polarization signal to a level were a detailed diagnostic of surface magnetic fields become feasible. But the trade off is the reduced interpretability of the retrieved mean line profile.

In an effort to provide the necessary analysis and diagnostic tools for the next generation of spectropolarimeter (PEPSI) at the $8.4 \mathrm{~m}$ Large Binocular Telescope (LBT) (Strassmeier et al., 2003, 2007), which, for the first time, provide the instrumental capabilities to detect and measure the magnetic fields of a significant number of active latetype stars, we have developed a novel multi-line reconstruction technique and a full Stokes Zeeman-Doppler imaging code. In the following we give a short presentation of the new line profile reconstruction technique as well as of our

\footnotetext{
^ e-mail: tcarroll@aip.de
}

new ZDI code IMap. In a first application we also present magnetic field surface maps of II Pegasi.

\section{The Zeeman-Doppler Imaging code}

The forward module of the ZDI code IMap incorporates the full numerical solution of the polarized radiative transfer equation by means of the so called Diagonal Lambda Operator method (DELO) (Rees et al., 1989). The code works on the basis of precalculated and/or interpolated Kurucz model atmospheres (Kurucz, 1993). Parameters for the individual spectral lines are retrieved from the Vald database (Piskunov et al., 1995) and line blends are full accounted for. The surface is parameterized on a variable equal-area or equal-degree partition with a minimum area of $1 \times 1$ degree. For each surface element a local Stokes vector is calculated with respect to its position and atmospheric parameters (Doppler velocity, bulk velocity, temperature, pressure, LOS magnetic field, micro- and macroturbulence). The atmospheric parameters are also allowed to vary along their local vertical direction which facilitates the complete description of a 3-dimensional atmosphere. Center-to-limb variations in the model atmosphere as well in the atmospheric parameters are fully account for by adjusting the depth stratification for each surface element with respect to the local reference frame of the observer. Field structures (in temperature and magnetic fields) can either be described by setting individual surface elements or using spherical harmonics.

The inverse module which is responsible for the fitting of the observed profiles by adjusting the model parameters implements two optimization algorithms. A LevenbergMarquardt method and a conjugate gradient method (Press et al. 
1992). Both algorithms are complemented by a regularization functional. We have used a similar maximum entropy regularization as formulated by Brown et al. (1991). which reads

$$
S=-\sum_{i}\left(\left|P_{i}\right|+\alpha\right) \log \frac{\left|P_{i}\right|+\alpha}{\left|m_{i}\right|+\alpha}-1,
$$

where the index $i$ runs over all surface elements, $\alpha$ is a small positive value to ensure that the entropy function is always greater than zero and $m_{i}$ is the so called default image of the surface element or segment. It is this default value which can give the entropy function a quite different characteristic. Even though the importance of the default images was never described in great detail in the ZDI literature the default images of the elements are usually set all to an equal value which is in most cases the global average of the parameter under consideration (i.e. magnetic field strength, inclination, azimuth or temperature). This is in fact problematic becuase this constrain is invariant under random mixing of the surface elements (pixels) and therefore inadequate for small scale (spatial) variations with great local differences in the parameters but also for smooth large scale variations in the parameters (Brown et al., 1991; Donati \& Brown, 1997; Pisk
2002). We therefore propose an adaptive local entropy function where the default images $m_{i}$ are retrieved by the actual mean value of the surrounding neighborhood pixels such that

$$
\begin{aligned}
S=-\sum_{i} & \left(\left|P_{i}\right|+\alpha\right) \\
& \times \log \frac{\left|P_{i}\right|+\alpha}{\frac{1}{k} \sum_{j=0}^{k} \beta(j)\left|m_{j}\right|+\alpha}-1 .
\end{aligned}
$$

Here the sum in the logarithm runs over the entire neighborhood of k elements and $\beta(j)$ is an extra term which includes a particular positve weighting. As the regularization term in the penalty function becomes important (compared to the regular squared error function) as soon as the fit of the individual Stokes profiles reaches the noise level of the observations, the default image is also the appropriate place to incorporate prior knowledge or assumptions that are not purely data driven (determined by the local gradient of the error function). We will described this topic in greater detail in a forthcoming paper.

The ZDI code can simultaneously or in a consecutive manner retrieve the temperature (Doppler imaging) and the magnetic field distribution (Zeeman Doppler imaging) by using the complete Stokes vector $(I, U, Q, V)$ or optionally only the Stokes $I$ and $V$ component.

\section{Multi-Line principal component reconstruction}

Most of the measured spectropolarimetric signals and profiles of individual spectral lines do not allow an in-depth analysis and interpretation which led to the development of powerful multi-line techniques such as LSD (Donati et al.,
1997). The success to which this method is able to extract and boost the signal quality comes at the expense of using approximate assumptions (weak fields, a-priori model atmospheres and Gaussian line profiles) and, moreover, of loosing the ability to use single spectral line profiles. Only a kind of mean line profile can be extracted by this technique which therefore has to be described in terms of artificial mean line parameters (e.g. excitation potential, Lande factor, oscillator strength etc.). In order to avoid simplified a-priori assumptions and making use of single spectral line profiles but benefiting at the same time from a multi-line cross-correlation technique we propose a multi-line principal component reconstruction technique. The basic idea is to use the redundancy and the variance in the observed data (the Stokes profiles) to determine a new coordinate system where each new coordinate axis accounts for a maximum of the variance in the original data. This can be conveniently expressed in terms of the covariance matrix $C_{\boldsymbol{x}}$ of the individual observed profile vectors $\boldsymbol{x}_{\boldsymbol{i}}$, which reads in the velocity domain

$$
\boldsymbol{C}_{\boldsymbol{x}}=\sum\left(\boldsymbol{x}_{n}(v)-\overline{\boldsymbol{x}}(v)\right)\left(\boldsymbol{x}_{n}(v)-\overline{\boldsymbol{x}}(v)\right)^{T},
$$

unov \& Kochukhov,

where $v=c \Delta \lambda / \lambda$ and $n$ is the number of individual spectral line profiles used for the analysis and $\overline{\boldsymbol{x}}$ is the mean Stokes profile of all spectral lines. The new set of coordinate axes which accounts for the maximum variance in the observed data (Stokes spectra) can then be determined by calculating the eigenvectors of the covariance matrix Eq. (3). This is exactly what the so called Principal Component Analysis (PCA) or Karhunen-Loeve transformation accomplishes (Bishop, 1995). We use the PCA method to decompose the entire set of observed Stokes spectra into a new coordinate system. This procedure will project the most coherent and systematic features in the observed Stokes profiles into the first few eigenvectors with the largest eigenvalues while the uncoherent features (i.e. noise) will be mapped to the less significant eigenvectors (with low eigenvalues). Since the covariance matrix Eq. (3) is symmetric it results in a set of orthogonal eigenvectors which we can used to decompose all observed Stokes spectra (with out any loss) into the new basis of eigenvectors $\boldsymbol{u}_{\boldsymbol{l}}$, as

$$
\boldsymbol{x}_{k}(v)=\sum_{l} \alpha_{k, l} \boldsymbol{u}_{l}(v)
$$

where $\alpha_{k, l}=\boldsymbol{x}_{k}(v) \boldsymbol{u}_{l}(v)$ is the scalar product (the projection or the cross-correlation) between the observed Stokes profile $\boldsymbol{x}_{k}(v)$ and the eigenvector $\boldsymbol{u}_{l}(v)$. If we make use of the well know decomposition and dimensionality reduction capabilities of the PCA method (Bishop, 1995) and using only the first few eigenvectors to reconstruct the original spectra $\boldsymbol{x}_{\boldsymbol{k}}$ such that

$$
\boldsymbol{x}_{k}(v)=\sum_{m<l} \alpha_{k, m} \boldsymbol{u}_{m}(v),
$$

we can reconstruct the individual Stokes profiles to an extent that the majority of the characteristic features of one 

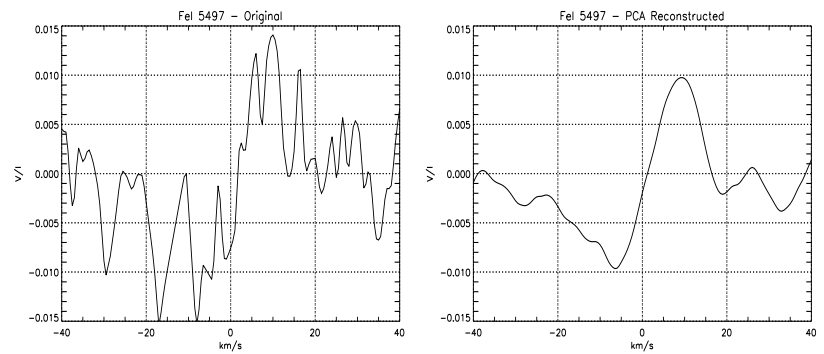

Fig. 1 On the left the original observed Stokes $V$ profile of the FeI $5497 \AA$ line and on the right side the multiline PCA reconstructed Stokes $V$ profile for one rotational phase.

particular line are well reproduced with a minimum of uncorrelated effects. This allows us to use a large set of observed Zeeman-sensitive spectral line profiles to get rid of the uncoherent effects like noise while retaining the common and systematic features (in all observed line profiles) which are induced by the Doppler- and Zeeman-effect. Since this method allows the reconstruction of a single line profile, all the known line parameter can be used in the following DI and ZDI process. In Fig. 1 we show a Stokes $V$ line profile for the iron line FeI $5497 \AA$ which has been observed for II Peg with the SOFIN spectrograph at the Nordic Optical Telescope (NOT) (Tuominen et al., 1999). For the multiline PCA reconstruction we have used 18 magnetic sensitive spectral lines (Fe I,Ca I , C I) in a wavelength range between 4600 and $6600 \AA$. To reconstruct the individual line profile we have used the three largest principal components. The Stokes $V$ line profile - although only 18 spectral lines in the covariance matrix are used - exhibit a much smoother and less noisy behavior and is due to the PCA analysis more reliable in terms of its interpretability. This method will be fully described and statistically analyzed in a forthcoming paper.

\section{The surface magnetic field of II Peg}

In a first application we have applied our code to spectropolarimetric observation of the K1 subgiant star II Peg observed with the SOFIN spectrograph at the NOT. The data (Stokes $I$ and Stokes $V$ ) were collected in an observing campaign in 2004 and cover a whole rotational period. The stellar parameters for the inversion are taken from Berdyugina e (1998). Prior to the actual Zeeman-Doppler inversion we have used our code in DI mode to retrieve the temperature distribution of the surface of II Peg, where we have used the CaI $6439 \AA$ A line. This information was subsequently used in the ZDI inversion to determine the magnetic field from the phase resolved Stokes $V$ profiles of the FeI $5497 \AA$ line, which was reconstructed by the multi-line PCA technique described in the preceeding section. After careful tests with different initialization and setups the observed profiles are well reproduced (see Fig 2). Despite the fact we have only

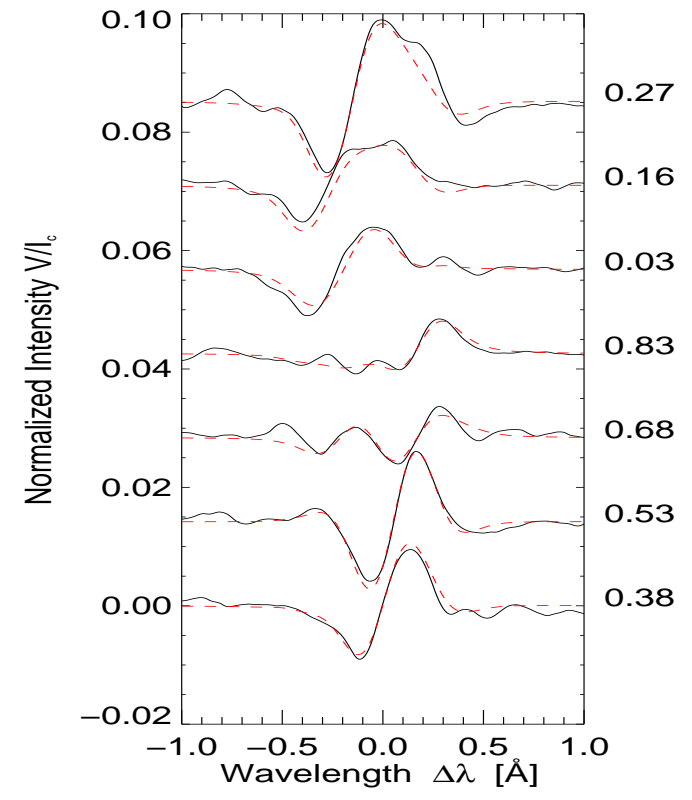

Fig. 2 Stokes $V$ profile fits (dashed) to the observed profiles (solid) of the iron line FeI $5497 \AA$ for different rotational phases.

used Stokes $V$ profiles the surface exhibits a rich magnetic surface structure as can be seen in Fig. 3 and Fig. 4. One of the conspicuous features in the ZDI map is that the magnetic activity is mainly located in one active longitude where the magnetic field shows a strong radial component. It will be interesting to see in forthcoming studies which expands over longer time periods if and in which way these regions of magnetic activity are associated with the active longitudes in the temperature distribution found by Berdyugina et al. (1999). As has been observed in former ZDI approaches

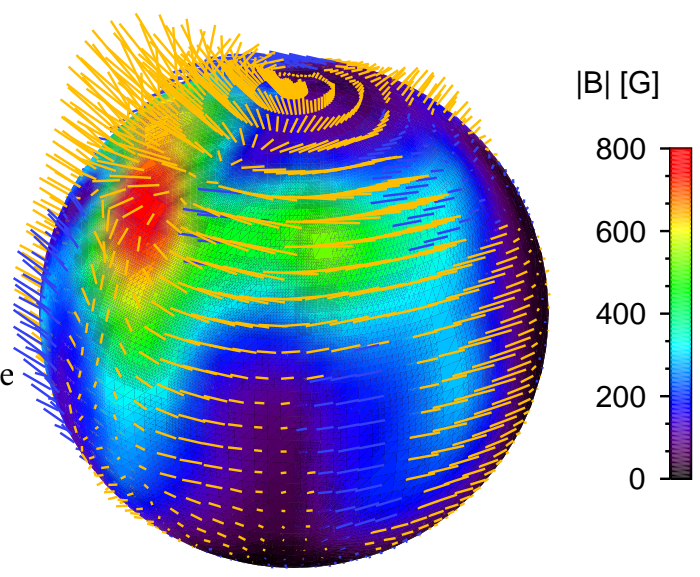

Fig. 3 Surface magnetic field of II Peg. The thin white lines indicating the field vector for each surface pixel and the underlying color gives the absolute field strength. 

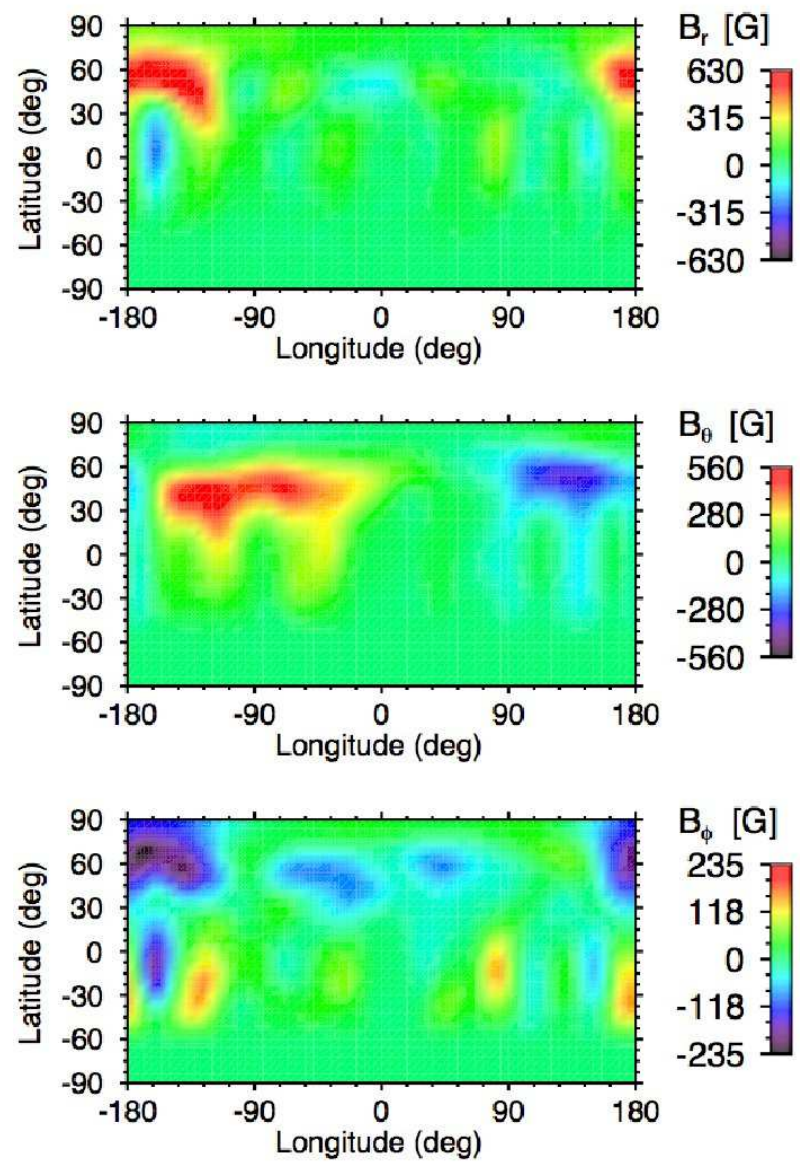

Fig. 4 Mercator plots of the surface magnetic field of II Peg. Top: the radial magnetic field; Middle: the azimuthal magnetic field component; Bottom : The meridional magnetic field component.

(Donati, 1999) we also notice only a small correlation between the dark and cool starspots as retrieved by our DI run and the magnetic field distribution from the ZDI. This seems to be plausible because of the strong suppression of the photon flux in the spot regions which are between 700 and $1000 \mathrm{~K}$ cooler than the quiet and hot parts of the surface. It should be mentioned that the retrieved surface field exhibit a strong imbalance in the magnetic polarity and we believe that this imbalance of magnetic flux results to a large extent from the inability of the circular polarization signal to capture the whole magnetic flux of the star, e.g. the probably strong magnetic fields in the spot regions of II Peg seem to be virtually absent in the Stokes $V$ profiles.

\subsection{Summary and conclusion}

We have presented a new Zeeman-Doppler imaging code which is particularly designed for the demands of retrieving surface temperature and magnetic field vector distributions of active late-type stars. Moreover, we have introduced a novel multi-line PCA reconstruction technique which relies on a minimal number of a-priori assumption and al- lows to extract and boost the signal-to-noise ratio of individual spectral line profiles. A first application shows the potential of the ZDI code and the new multi-line technique. Even though the field structure results in a smooth distribution it should be noted that the reconstruction of the surface magnetic field is far from being a well posed problem. Small differences in the underlying initialization, in the regularization function or the temperature distribution as well as changes in the optimization strategy can lead to rather drastic effects in the recovered magnetic surface fields (see forthcoming paper of Carroll et al. 2008)). It is therefore of particular interest to make the linear polarization profiles available and to retrieve circular polarized profiles with the best possible accuracy and signal-to-noise ratio to provide more constrains to the solution of the ZDI inversion and to reveal more of the hidden magnetic field information in the spectra.

\section{References}

Berdyugina, S. V., Jankov, S., Ilyin, I., Tuominen, I., \& Fekel, F. C. 1998, A\&A, 334, 863

Berdyugina, S. V., Berdyugin, A. V., Ilyin, I., \& Tuominen, I. 1999, A\&A, 350, 626

Bishop, C.M., 1995, Neural Networks for Pattern Recogniti on, Oxford University Press

Brown, S. F., Donati, J.-F., Rees, D. E., \& Semel, M. 1991, A\&A, 250, 463

Donati, J.-F., Semel, M., Carter, B. D., Rees, D. E., \& Collier Cameron, A. 1997, MNRAS, 291, 658

Donati, J.-F., \& Brown, S. F. 1997, A\&A, 326, 1135

Donati, J.-F. 1999, Solar and Stellar Activity: Similarities and Differences, 158, 27

Donati, J.-F. 2003, Astronomical Society of the Pacific Conference Series, 307, 41

Kurucz, R. 1993, ATLAS9 Stellar Atmosphere Programs and $2 \mathrm{~km} / \mathrm{s}$ grid. Kurucz CD-ROM No. 13. Cambridge, Mass.: Smithsonian Astrophysical Observatory, 1993., 13 ,

Petit, P. 2006, Astronomical Society of the Pacific Conference Series, 358, 335

Piskunov, N. E., Kupka, F., Ryabchikova, T. A., Weiss, W. W., \& Jeffery, C. S. 1995, A\&A suppl., 112, 525

Piskunov, N., \& Kochukhov, O. 2002, A\&A, 381, 736

Press, W. H., Teukolsky, S. A., Vetterling, W. T., \& Flannery, B. P. 1992, Cambridge: University Press, —c1992, 2nd ed.,

Rees, D. E., Durrant, C. J., \& Murphy, G. A. 1989, ApJ, 339,1093

Semel, M. 1989, A\&A, 225, 456

Semel, M., \& Li, J. 1996, Solar Physics, 164, 417

Strassmeier, K. G., Hofmann, A., Woche, M. F., Rice, J. B., Keller, C. U., Piskunov, N. E., \& Pallavicini, R. 2003, SPIE, 4843, 180

Strassmeier, K. G., Woche, M., Andersen, M., \& Ilyin, I. 2007, Astronomische Nachrichten, 328, 627 
Tuominen, I., Ilyin, I., \& Petrov, P. 1999, Astrophysics with the NOT, 47 